\title{
Function Theoretic Properties of Symmetric Powers of Complex Manifolds
}

\author{
Włodzimierz Zwonek ${ }^{1}$
}

Received: 2 April 2019 / Published online: 4 July 2019

(c) The Author(s) 2019

\begin{abstract}
In the paper we study properties of symmetric powers of complex manifolds. We investigate a number of function theoretic properties [e.g. (quasi) $c$-finite compactness, existence of peak functions] that are preserved by taking the symmetric power. The case of symmetric products of planar domains is studied in a more detailed way. In particular, a complete description of the Carathéodory and Kobayashi hyperbolicity and Kobayashi completeness in that class of domains is presented.
\end{abstract}

Keywords Symmetric power of complex manifolds · (quasi) $c$-finite compactness · Peak functions - Symmetrized polydisc $\cdot$ Kobayashi and Carathéodory hyperbolicity (completeness)

Mathematics Subject Classification $32 \mathrm{C} 15 \cdot 32 \mathrm{~T} 40 \cdot 32 \mathrm{~F} 45$

\section{Introduction}

Let $X$ be a connected complex manifold of dimension $m$. We define its $n$ - $t$ h symmmetric power $X_{\text {sym }}^{n}$ as the quotient $X^{n}$ under the action of the group of all permutations of $\{1, \ldots, n\}$. Recall that $X_{\text {sym }}^{n}$ has the structure of a complex analytic space. In the case when $m=1$ the space $X_{\text {sym }}^{n}$ is actually a complex manifold. If $X=D \subset \mathbb{C}$ is a domain then we have a realization of $D_{\text {sym }}^{n}$ as a domain in $\mathbb{C}^{n}$. More precisely, its biholomorphic realization is the following $n$-dimensional symmetrization (or symmetric product of planar domains)

$$
S_{n}(D):=\pi_{n}\left(D^{n}\right)
$$

where $\pi_{n}: \mathbb{C}^{n} \mapsto \mathbb{C}^{n}$ is the symmetrization map (the $j$-th coordinate is the $j$-th elementary symmetric polynomial). In other words $\pi_{n, j}\left(\lambda_{1}, \ldots, \lambda_{n}\right)=\sigma_{j}\left(\lambda_{1}, \ldots, \lambda_{n}\right)$,

Włodzimierz Zwonek

wlodzimierz.zwonek@uj.edu.pl

1 Institute of Mathematics, Faculty of Mathematics and Computer Science, Jagiellonian University, Łojasiewicza 6, 30-348 Kraków, Poland 
$\lambda_{j} \in \mathbb{C}, j=1, \ldots, n$, where $\sigma_{j}$ satisfies the equality

$$
\left(\lambda-\lambda_{1}\right) \cdots\left(\lambda-\lambda_{n}\right)=\lambda^{n}+\sum_{j=1}^{n}(-1)^{j} \sigma_{j}\left(\lambda_{1}, \ldots, \lambda_{n}\right) \lambda^{n-j}, \lambda \in \mathbb{C} .
$$

As to the background on basic properties of symmetric powers we refer the Reader e. g. to [18].

\subsection{Description of Results}

In the paper we present a number of properties of $X_{\mathrm{sym}}^{n}$. Some of them may be obtained from general properties of the realization of $\mathbb{D}_{\mathrm{sym}}^{n}$ as a domain, i. e. the so called the symmetrized polydisc $\mathbb{G}_{n}:=S_{n}(\mathbb{D})$, $(\mathbb{D}$ denotes the unit disc in $\mathbb{C})$. The last domain has been extensively studied in the last two decades (see for instance $[1,5,7,9]$ and references there).

The starting point for considerations in the paper was inspired by recent developments on the function theory in symmetric powers (see e. g. [2,3] and [4]).

First we present a more general result to that of Theorem 1.4 in [2] where the proof of the Kobayashi completeness of symmetric powers of some of Riemann surfaces relies on the proof of existence of peak functions. Similarly as in [2] the presentation below actually deals with a stronger version of completeness-the $c$-finite compactness and shows that the notion (more precisely, the weaker notion of quasi $c$-finite compactness) is preserved under taking the symmetric power, which is done in a general case of complex manifolds (Theorem 1). Following the same line of argument relying upon analoguous results in the symmetrized polydisc, we present a result on the existence of peak functions in symmetric powers (Theorem 6).

In Sect. 3, we concentrate on properties of symmetric products of planar domains in $\mathbb{C}$. We show the linear convexity of such domains (Proposition 9), we present a Riemann-type mapping theorem for them (Theorem 12) and then we discuss to which extent the Kobayashi hyperbolicity (completeness) is preserved under taking the symmetric power-a complete description of Kobayashi hyperbolicity (completeness) in that class is given in Theorem 16. Finally, we present a result on preserving the Carathéodory hyperbolicity under taking the symmetric powers of planar domains (Proposition 18).

\section{General Case}

In this Section, we present results for a general class of symmetric powers of manifolds.

\section{1 (Quasi) c-Finite Compactness}

Let us recall that any holomorphic mapping $f: X \rightarrow Y$ ( $X$ and $Y$ are complex manifolds, not necessarily of the same dimension) induces a holomorphic map- 
ping $F_{f}: X_{\text {sym }}^{n} \rightarrow Y_{\text {sym }}^{n}$ by the formula [a typical element of $X_{\text {sym }}^{n}$ generated by $\left(z_{1}, \ldots, z_{n}\right) \in X^{n}$ is denoted by $\left.\left\langle z_{1}, \ldots, z_{n}\right\rangle\right]$

$$
F_{f}\left(\left\langle z_{1}, \ldots, z_{n}\right\rangle\right):=\left\langle f\left(z_{1}\right), \ldots, f\left(z_{n}\right)\right\rangle, z_{j} \in X, j=1, \ldots, n .
$$

In the case $Y=\mathbb{D}$, we have the holomorphicity of the mapping

$$
X_{\text {sym }}^{n} \ni\left\langle z_{1}, \ldots, z_{n}\right\rangle \rightarrow \pi_{n}\left(f\left(z_{1}\right), \ldots, f\left(z_{n}\right)\right\rangle \in \mathbb{G}_{n}
$$

We should also be aware of the fact that any holomorphic function $F: X_{\text {sym }}^{n} \rightarrow \mathbb{D}$ may be identified with a symmetric function $\tilde{F}: X^{n} \rightarrow \mathbb{D}$ - a function $\tilde{F} \in \mathcal{O}\left(X^{n}, \mathbb{D}\right)$ is called symmetric if $\tilde{F}\left(z_{\sigma(1)}, \ldots, z_{\sigma(n)}\right)=\tilde{F}\left(z_{1}, \ldots, z_{n}\right)$ for any permutation $\sigma$ of $\{1, \ldots, n\}, z_{1}, \ldots, z_{n} \in X$. The identification is given by the relation

$$
F\left(\left\langle z_{1}, \ldots, z_{n}\right\rangle\right)=\tilde{F}\left(z_{1}, \ldots, z_{n}\right), z_{j} \in X, ; j=1, \ldots, n .
$$

The space of symmetric holomorphic functions $X^{n} \rightarrow \mathbb{D}$ is denoted by $\mathcal{O}_{s}\left(X^{n}, \mathbb{D}\right)$. lows:

The last observation lets us define the Carathéodory pseudodistance $c_{X_{\mathrm{sym}}^{n}}$ as fol-

$$
\begin{aligned}
& c_{X_{\text {sym }}^{n}}\left(\left\langle z_{1}, \ldots, z_{n}\right\rangle,\left\langle w_{1}, \ldots, w_{n}\right\rangle\right) \\
& \quad=\sup \left\{p\left(0, F\left(w_{1}, \ldots, w_{n}\right)\right): F \in \mathcal{O}_{s}\left(X^{n}, \mathbb{D}\right), F\left(z_{1}, \ldots, z_{n}\right)=0\right\},
\end{aligned}
$$

where $p$ is the Poincare distance on $\mathbb{D}$.

If, on some complex structure $X$ (e. g. a complex manifold), we may well-define the Carathéodory pseudodistance we call $X$ quasi $c$-finitely compact if for any sequence $\left(z_{k}\right)_{k} \subset X$ without the accummulation point we have $c_{X}\left(z_{1}, z_{k}\right) \rightarrow \infty$. Recall that if $X$ is additionally Carathéodory hyperbolic, i. e. $c_{X}(w, z)>0, w, z \in X, w \neq z$ then $X$ is called $c$-finitely compact. As to the basic properties related to the Carathéodory pseudodositance (as well as to other holomorphically invariant functions) we refer the Reader to e. g. $[9,11]$.

As we shall see below a natural property that is inherited by the symmetric power is the quasi $c$-finite compactness.

Theorem 1 Let $X$ be a connected complex manifold. Then $X_{\mathrm{sym}}^{n}$ is quasi c-finitely compact iff $X$ is quasi c-finitely compact.

Proof Assume that $X$ is quasi $c$-finitely compact. Fix $\left\langle z_{1}, \ldots, z_{n}\right\rangle \in X_{\text {sym }}^{n}$. Let $\left(\left\langle w_{1}^{k}, \ldots, w_{n}^{k}\right\rangle\right)_{k} \subset X_{\text {sym }}^{n}$ be a sequence without an accummulation point. Then without loss of generality we may assume that $\left(w_{1}^{k}\right)_{k}$ has no accummulation point in $X$. Let $f_{k} \in \mathcal{O}(X, \mathbb{D})$ be such that $f_{k}\left(z_{1}\right)=0$ and $f_{k}\left(w_{1}^{k}\right) \rightarrow 1$. Then the contractivity of the Carathéodory pseudodistance gives the following

$$
\begin{aligned}
& c_{X_{\mathrm{sym}}^{n}}\left(\left\langle z_{1}, \ldots, z_{n}\right\rangle,\left\langle w_{1}^{k}, \ldots, w_{n}^{k}\right\rangle\right) \\
& \quad \geq c_{\mathbb{G}_{n}}\left(\pi_{n}\left(f_{k}\left(z_{1}\right), \ldots, f_{k}\left(z_{n}\right)\right), \pi_{n}\left(f_{k}\left(w_{1}^{k}\right), \ldots, f_{k}\left(w_{n}^{k}\right)\right) .\right.
\end{aligned}
$$


Since $\pi_{n}\left(f_{k}\left(w_{1}^{k}\right), \ldots, f_{k}\left(w_{n}^{k}\right)\right) \rightarrow \partial \mathbb{G}_{n}$ and the set $\left\{\pi_{n}\left(f_{k}\left(z_{1}\right), \ldots, f_{k}\left(z_{n}\right)\right): k=\right.$ $1,2, \ldots\}$ is relatively compact in $\mathbb{G}_{n}$, the $c$-finite compactness of $\mathbb{G}_{n}$ (see [15]) gives the convergence of the above expression to infinity which finishes the proof.

Assume now that $X_{\text {sym }}^{n}$ is quasi $c$-finitely compact. Fix $\left\langle z_{1}, \ldots, z_{n}\right\rangle \in X_{\text {sym }}^{n}$. Let $\left(w_{1}^{k}\right)_{k} \subset X$ be a sequence without accumulation point. Then the sequence $\left(\left\langle w_{1}^{k}, z_{2}, \ldots, z_{n}\right\rangle\right)_{k}$ has no accumulation point, either. Then the inequalities

$$
c_{X}\left(z_{1}, w_{1}^{k}\right) \geq c_{X_{\mathrm{sym}}^{n}}\left(\left\langle z_{1}, \ldots, z_{n}\right\rangle,\left\langle w_{1}^{k}, z_{2}, \ldots, z_{n}\right\rangle\right) \rightarrow \infty
$$

show the quasi $c$-finite compactness of $X$.

Remark 2 In the case when $X$ is a bounded domain in $\mathbb{C}$ the above theorem is a reformulation of Theorem 4.1 in [12] (applied to the proper holomorphic mapping $\left(\pi_{n}\right)_{\mid D^{n}}: D^{n} \rightarrow S_{n}(D)$ ). We also should be aware of the fact that the idea of the proof of the above theorem is exactly the same as that of Theorem 4.1 in [12].

Remark 3 In Theorem 1.4 in [2] a result on Kobayashi completeness of symmetric powers of some Riemann surfaces is formulated. The proof relies on the existence of some peak functions together with the application of Result 3.6 from [2], in which the fact of $c$-finite compactness is claimed under assumption of the existence of some peak functions. It is however not explained us how the necessary fact of the Carathéodory hyperbolicity is obtained only with the help of the existence of peak functions (in the case studied in the reasoning from [11], to which the paper [2] appeals, the hyperbolicity is trivially satisfied).

Remark 4 If $d$ denotes a family of holomorphically invariant functions (for instance the Carathéodory ( $c$ ) or Kobayashi $(k)$ pseudodistance) then $d$-hyperbolicity of a complex manifold $X_{\text {sym }}^{n}$ implies the $d$-hyperbolicity of $X$. Actually, fix $w_{1}, z_{1} \in X, w_{1} \neq z_{1}$. Choose $w_{2} \in X$. Then $\left\langle w_{1}, w_{2}, \ldots, w_{2}\right\rangle \neq\left\langle z_{1}, w_{2}, \ldots, w_{2}\right\rangle$. Consequently,

$$
c_{X}\left(w_{1}, z_{1}\right) \geq c_{X_{\text {sym }}^{n}}\left(\left\langle w_{1}, w_{2}, \ldots, w_{2}\right\rangle,\left\langle z_{1}, w_{2}, \ldots, w_{2}\right\rangle\right)>0
$$

As to the implication:

$X$ is $d$-hyperbolic $\Longrightarrow X_{\text {sym }}^{n}$ is $d$-hyperbolic

the observation in the next remark shows that it is not true in general.

Remark 5 It would be tempting to formulate a similar equivalence as in Theorem 1 for the notion of the Kobayashi quasi completeness. However, the example $\mathbb{C} \backslash\{0,1\}$ shows that the Kobayashi completeness of $X$ does not guarantee any reasonable property of the Kobayashi pseudodistance of $X_{\text {sym }}^{n}$.

In fact, put $D:=\mathbb{C} \backslash\{0,1\}, n=2$. Then

$$
S_{2}(D)=\mathbb{C}^{2} \backslash(\mathbb{C} \times\{0\} \cup\{(\lambda+1, \lambda): \lambda \in \mathbb{C}\})
$$

The last is the space $\mathbb{C}^{2}$ with two complex lines intersected which is affinely isomorphic with $\mathbb{C}_{*}^{2}$ for which the Kobayashi pseudodistance vanishes. In the sequel we shall 
present a complete description of the Kobayashi hyperbolicity, Kobayashi completeness, Carathéodory hyperbolicity and $c$-finite compactness in the class of symmetric products of planar domains.

\subsection{Peak Functions}

In the paper [2], the proof of the Kobayashi completeness (Theorem 1.4) was conducted with the help of the existence of peak functions (that was done for some Riemann surfaces). We generalize the result and simplify the proof below. We also see that we may reduce the proof of the existence of peak functions in symmetric powers to the existence of some of peak functions in the original complex manifold.

For a domain $Y$ in a complex manifold $X$ and $K \subset \bar{Y}$ we define $A(Y, K):=$ $\mathcal{O}(Y) \cap \mathcal{C}(Y \cup K)$. We call a point $z \in K$ an $A(Y, K)$ peak point if there is an $f \in A(Y, K)$ such that $|f|<1$ on $Y$ and $f(z)=1$. We call $f$ the $A(Y, K)$ peak function at $z$.

Theorem 6 Let $Y$ be a domain in a complex manifold $X$. Assume that $z_{1} \in \partial Y$ is the $A\left(Y,\left\{z_{1}, \ldots, z_{n}\right\}\right)$ peak point, where $z_{2}, \ldots, z_{n} \in \bar{Y}$. Then the point $\left\langle z_{1}, \ldots, z_{n}\right\rangle$ is an $A\left(Y_{\mathrm{sym}}^{n},\left\{\left\langle z_{1}, \ldots, z_{n}\right\rangle\right\}\right)$ peak point.

Proof Let $f$ be an $A\left(Y,\left\{z_{1}, \ldots, z_{n}\right\}\right)$ peak function at $z_{1}$. Let $F \in \mathcal{O}\left(\mathbb{G}_{n} \cup U\right)$, where $U$ is a neighborhood of $\pi_{n}\left(f\left(z_{1}\right), \ldots, f\left(z_{n}\right)\right) \in \partial G_{n}$ be such that

$$
F\left(\pi_{n}\left(f\left(z_{1}\right), \ldots, f\left(z_{n}\right)\right)\right)=1 \text { and }|F|<1 \text { on } \mathbb{G}_{n}
$$

(see Theorem 2.1 in [12]). The function

$$
Y_{\text {sym }}^{n} \cup\left\{\left\langle z_{1}, \ldots, z_{n}\right\rangle\right\} \ni\left\langle w_{1}, \ldots, w_{n}\right\rangle \rightarrow F\left(\pi_{n}\left(f\left(w_{1}\right), \ldots, f\left(w_{n}\right)\right)\right)
$$

is the desired peaking function.

Remark 7 The assumption in Theorem 6 is a weaker one than that in the proof of Theorem 1.4 of [2].

\section{Remarks on Symmetric Products of Planar Domains}

In this section we present properties of symmetric products of planar domains.

\subsection{General Properties}

Recall that if $D$ is a domain in $\mathbb{C}$ then we have a nice representation of $D_{\text {sym }}^{n}$ as a domain in $\mathbb{C}$. We work therefore on this representation, i. e. the domain $S_{n}(D)$. First we collect some facts concerning $S_{n}(D)$. Below we list some known or straightforward properties of $S_{n}(D)$.

Remark $8 \cdot S_{n}(D)$ is a domain in $\mathbb{C}^{n}$ and $\left(\pi_{n}\right)_{\mid D^{n}}: D^{n} \rightarrow S_{n}(D)$ is proper onto the image, 
- if $\Sigma_{n}:=\left\{z \in D^{n}: z_{j}=z_{k}\right.$ for some $\left.j \neq k\right\}$ then $\pi_{n}: D^{n} \backslash \Sigma_{n} \rightarrow S_{n}(D) \backslash \pi_{n}\left(\Sigma_{n}\right)$ is a holomorphic covering,

- $S_{n}(D)$ is bounded iff $D$ is bounded,

- $\overline{S_{n}(D)}=\pi_{n}\left(\bar{D}^{n}\right), \partial S_{n}(D)=\pi_{n}\left(\partial D \times \bar{D}^{n-1}\right)$,

- if $D$ is additionally bounded then the mapping $\left(\pi_{n}\right)_{D^{n}}$ maps $A\left(D^{n}\right)$ peak points $(A(\Omega):=\mathcal{O}(\Omega) \cap \mathcal{C}(\bar{\Omega}))$ onto $A\left(S_{n}(D)\right)$ peak points. In particular, the Shilov boundary $\partial_{S}\left(S_{n}(D)\right)$ equals $S_{n}\left(\partial_{S}(D)\right)$ (see Theorem 3.1 in [12]).

Recall that a domain $\Omega \subset \mathbb{C}^{n}$ is called linearly convex if for any $w \in \mathbb{C}^{n} \backslash \Omega$ we may find an affine hyperplane $H$ passing through $w$ and disjoint from $\Omega$. Following step by step the idea from [15] we get the linear convexity of $S_{n}(D)$.

Proposition 9 Let $D$ be a domain in $\mathbb{C}, n \geq 2$. Let $w=\pi_{n}(\mu) \in \mathbb{C}^{n} \backslash S_{n}(D)$ be such that $\mu_{1} \in \mathbb{C} \backslash D$. Then the affine hyperplane

$$
\begin{aligned}
& H\left(w, \mu_{1}\right) \\
& \quad:=\left\{\left(\mu_{1}+z_{1}, \mu_{1} z_{1}+z_{2}, \ldots, \mu_{1} z_{n-2}+z_{n-1}, \mu_{1} z_{n-1}\right):\left(z_{1}, \ldots, z_{n-1}\right) \in \mathbb{C}^{n-1}\right\}
\end{aligned}
$$

passes through $w$ and is disjoint from $S_{n}(D)$. In particular, $S_{n}(D)$ is linearly convex.

Proof Note that

$$
\begin{aligned}
& H\left(w, \mu_{1}\right) \\
& =\left\{\left(\mu_{1}+\pi_{n-1,1}\left(\lambda^{\prime}\right), \mu_{1} \pi_{n-1,1}\left(\lambda^{\prime}\right)\right.\right. \\
& \left.\left.\quad+\pi_{n-1,2}\left(\lambda^{\prime}\right), \ldots, \mu_{1} \pi_{n-1, n-1}\left(\lambda^{\prime}\right)\right): \lambda^{\prime} \in \mathbb{C}^{n-1}\right\},
\end{aligned}
$$

which follows from the surjectivity of the mapping $\pi_{n-1}: \mathbb{C}^{n-1} \rightarrow \mathbb{C}^{n-1}$. It follows that $H\left(w, \mu_{1}\right)$ is disjoint from $S_{n}(D)$. Substituting $\lambda^{\prime}=\left(\mu_{2}, \ldots, \mu_{n}\right)$ we see that $w=\pi_{n}\left(\mu_{1}, \ldots, \mu_{n}\right) \in H\left(w, \mu_{1}\right)$, which finishes the proof.

Remark 10 Let us draw our attention to the following property. If $\mu_{1}, \ldots, \mu_{k} \in \mathbb{C}$ $(1 \leq k \leq n)$ then the set

$$
\begin{aligned}
& H\left(\mu_{1}, \ldots, \mu_{k}\right) \\
& \quad:=\left\{\pi_{n}\left(\mu_{1}, \ldots, \mu_{k}, \lambda_{1}, \ldots, \lambda_{n-k}\right): \lambda_{j} \in \mathbb{C}, j=1, \ldots, n-k\right\}
\end{aligned}
$$

is an $(n-k)$-dimensional affine space (in the proof of the previous proposition we considered the case $k=1)$. Actually, first note that the mapping $\psi:=\pi_{n}\left(\mu_{1}, \ldots, \mu_{k}, \cdot\right)$ : $\mathbb{C}^{n-k} \rightarrow \mathbb{C}^{n}$ is proper. Additionally, the form of $\pi_{n}$ easily implies that $\psi$ is an affine mapping of variables $\pi_{n-k}\left(\lambda_{1}, \ldots, \lambda_{n-k}\right)$ and $\pi_{n-k}: \mathbb{C}^{n-k} \rightarrow \mathbb{C}^{n-k}$ is also onto. All these facts give the desired property of $H\left(\mu_{1}, \ldots, \mu_{k}\right)$.

We show how some properties of $D$ induce the same ones of $S_{n}(D)$ (compare Theorem 1). The first notion that we discuss is the hyperconvexity. 
Proposition 11 Let $D$ be a domain in $\mathbb{C}, n \geq 2$. Then $S_{n}(D)$ is hyperconvex iff $D$ is hyperconvex.

Proof Let $D$ be hyperconvex and let $u: D \rightarrow(-\infty, 0)$ be a negative subharmonic exhaustion function. Define

$$
v(z):=\max \left\{u\left(w_{j}\right): \pi_{n}\left(w_{1}, \ldots, w_{n}\right)=z, j=1, \ldots, n\right\}, z \in S_{n}(D) .
$$

The properness of $\left(\pi_{n}\right)_{D^{n}}$ onto the image and the geometry of $S_{n}(D)$ imply that $v$ is a negative plurisubharmonic exhaustion function of $S_{n}(D)$.

To prove the opposite implication fix some $\lambda_{1}, \ldots, \lambda_{n-1} \in D$ and let $v$ be the negative plurisubharmonic exhaustion function on $S_{n}(D)$. Let $u(\cdot):=$ $v\left(\pi_{n}\left(\lambda_{1}, \ldots, \lambda_{n-1}, \cdot\right)\right)$ be defined on $D$. Then $u$ is a negative exhaustion subharmonic function on $D$.

\subsection{Riemann-Type Mapping Theorem}

For a domain $\Omega \subset \mathbb{C}^{n}$ we define the Lempert function as follows

$$
l_{\Omega}(w, z):=\inf \{p(0, \sigma): \exists f \in \mathcal{O}(\mathbb{D}, \Omega) \text { such that } f(0)=w, f(\sigma)=z\} .
$$

Recall that the Lempert Theorem (see e. g. $[9,13]$ ) states that if $\Omega$ is convex then $l_{\Omega} \equiv c_{\Omega}$.

In the next result we show a Riemann-type mapping theorem for symmetric powers of planar domains.

Theorem 12 Let $D$ be a bounded, hyperconvex domain in $\mathbb{C}, n \geq 2$. Assume that $c_{S_{n}(D)} \equiv l_{S_{n}(D)}$. Then $D$ is biholomorphic to $\mathbb{D}$ and $n=2$.

Proof Choose pairwise distinct points $\lambda_{1}^{0}, \ldots, \lambda_{n-1}^{0} \in \partial$ (int $\bar{D}$ ) (the fact that $D$ is bounded allows us to make such a choice) and two distinct points $\lambda_{n}^{0}, \mu_{n}^{0} \in D$. Let us also choose sequences $D \ni \lambda_{j}^{k} \rightarrow_{k \rightarrow \infty} \lambda_{j}^{0}, j=1, \ldots, n-1$. Now the equality between the Lempert function and the Carathéodory distance and the tautness of $S_{n}(D)$ imply that there exist holomorphic mappings

$$
f_{k}: \mathbb{D} \rightarrow S_{n}(D), F_{k}: S_{n}(D) \rightarrow \mathbb{D}
$$

such that $F_{k} \circ f_{k}=\operatorname{id}_{\mathbb{D}}$ and $f_{k}(0)=\pi_{n}\left(\lambda_{1}^{k}, \ldots, \lambda_{n-1}^{k}, \lambda_{n}^{0}\right), f_{k}\left(\sigma_{k}\right)=\pi_{n}\left(\lambda_{1}^{k}, \ldots\right.$, $\left.\lambda_{n-1}^{k}, \mu_{n}^{0}\right)$, where $\sigma_{k} \in(0,1)$.

Define

$$
G_{k}(\lambda):=F_{k}\left(\pi_{n}\left(\lambda_{1}^{k}, \ldots, \lambda_{n-1}^{k}, \lambda\right)\right), \lambda \in D .
$$

Without loss of generality (taking if necessary a subsequence) we have the following convergences (we use here the boundedness of $D$ ):

$$
\sigma_{k} \rightarrow \sigma_{0} \in(0,1), f_{k} \rightarrow f \text { and } G_{k} \rightarrow G \text { locally uniformly, }
$$


where $f: \mathbb{D} \rightarrow \overline{S_{n}(D)}, G: D \rightarrow \mathbb{D}, f(0)=\pi_{n}\left(\lambda_{1}^{0}, \ldots, \lambda_{n}^{0}\right), f(\sigma)=$ $\pi_{n}\left(\lambda_{1}^{0}, \ldots, \lambda_{n-1}^{0}, \mu_{n}^{0}\right), G\left(\lambda_{n}^{0}\right)=0, G\left(\mu_{n}^{0}\right)=\sigma$.

Define $\tilde{g}$ (respectively, $\tilde{g}_{k}$ ) to be the $n$ components of the multivalued function $\pi_{n}^{-1} \circ f$ (respectively, $\pi_{n}^{-1} \circ f_{k}$ ). We know that all the components of $\tilde{g}$ (respectively, $\tilde{g}_{k}$ ) have values in $\bar{D}$ (respectively, $D$ ). Additionally at the points 0 and $\sigma$ all but one components of $\tilde{g}$ are from $\partial D$. Note that the values of $f$ at these two points are regular values for the proper holomorphic mapping $\pi_{n}$ and thus the functions $\pi_{n}^{-1} \circ f$ near these two points $(0$ and $\sigma)$ may be chosen to be a holomorphic mapping.

The openness of holomorphic functions and the description of the closure of $\pi_{n}(D)$ together with the fact that $\lambda_{j}^{0} \in \partial$ (int $\left.\bar{D}\right), j=1, \ldots, n-1$, imply that near these two points all but one components of $\tilde{g}$ are constant (and equal to $\lambda_{1}^{0}, \ldots, \lambda_{n-1}^{0}$ ) whereas the last one is from $D$. The fact that some nonempty open part of $f(\mathbb{D})$ is lying in the complex line $L:=\left\{\pi_{n}\left(\lambda_{1}^{0}, \ldots, \lambda_{n-1}^{0}, \lambda\right): \lambda \in \mathbb{C}\right\}$ implies easily that $f(\mathbb{D}) \subset L$ and consequently all but one components of $\tilde{g}$ are constant (equal to $\lambda_{j}^{0}$, $j=1, \ldots, n-1)$ and the last component is a nonconstant holomorpic function $g: \mathbb{D} \rightarrow$ int $(\bar{D})$ with $g(0)=\lambda_{n}^{0}$ and $g(\sigma)=\mu_{n}^{0}$. We show below that we have even more; namely, $g(\mathbb{D}) \subset D$. In fact, following the same line of argument we get more; the multivalued functions $\tilde{g}_{k}$ have the following property: there exists a sequence $r_{k} \rightarrow 1$, $0<r_{k}<1$, such that the multivalued functions $\tilde{g}_{k}$ are actually holomorphic functions when restricted to $r_{k} \mathbb{D}$ with values in $D$. Moreover, taking the last component of the multifunction $\tilde{g}_{k}$ (which we denote by $g_{k}$ ) we get that $g_{k} \rightarrow g$ locally uniformly on $\mathbb{D}$. Recall that $g_{k}: r_{k} \mathbb{D} \rightarrow D$ so the Hurwitz theorem implies that $g(\mathbb{D}) \subset D$.

Since $G \circ g(0)=0, G \circ g(\sigma)=\sigma$, the Schwarz Lemma implies that $G \circ g$ is the identity. Consequently, $D$ is biholomorphic to $\mathbb{D}$ (with biholomorphisms given by $g$ or $G$ ). The results on the symmetrized polydisc (see $[1,5,14]$ ) imply that $n=2$.

Remark 13 It would be interesting to see whether some analogue of the Lempert theorem or the rigidity of the group of automorphims holds for $\left(\mathbb{B}_{m}\right)_{\text {sym }}^{n}, m, n \geq 2$ (compare $[1,4,5,7,14]$ ). It is also interesting to which extent we could relax assumptions in Theorem 12. Recall that without some extra assumptions we cannot hope for the implication:

$$
l_{S_{n}(D)} \equiv c_{S_{n}(D)} \Longrightarrow l_{D} \equiv c_{D} \text {. }
$$

Namely, in the example $D:=\mathbb{C} \backslash\{0,1\}$ we have the identities $l_{S_{2}(D)} \equiv c_{S_{2}(D)} \equiv 0$ whereas $c_{D} \equiv 0$ and $l_{D}(w, z)>0, w \neq z$.

\subsection{Kobayashi Hyperbolicity and Completeness of Symmetric Products of Planar Domains}

Recall that the Kobayashi (pseudo)distance $k_{\Omega}$ of a domain $\Omega \subset \mathbb{C}^{n}$ may be defined as the largest pseudodistance smaller than or equal to $l_{\Omega}$. The domain $\Omega$ is called Kobayashi hyperbolic if $k_{\Omega}$ is a distance. If additionally, $\left(\Omega, k_{\Omega}\right)$ is a complete metric space then $\Omega$ is called Kobayashi complete. Recall that the Kobayashi completeness of a Kobayashi hyperbolic domain is equivalent to the $k$-finite compactness, i. e. the fact that $k_{\Omega}\left(z, z^{k}\right) \rightarrow \infty$ for some (any) $z \in \Omega$ and any sequence $\left(z^{k}\right)_{k} \subset \Omega$ having no accummulation point (see e. g. [9,11]). 
We already know that representations of symmetric products of planar domains are linearly convex. It is worth mentioning that a bounded linearly convex domain $\Omega \subset \mathbb{C}^{n}$ is automatically Kobayashi complete-as mentioned by N. Nikolov to the author it follows directly from Lemma 3.3 in [16].

Proposition 14 (see Lemma 3.3 in [16]) Let $\Omega \subset \mathbb{C}^{n}$ be a bounded linearly convex domain. Then $\Omega$ is Kobayashi complete.

The above proposition allows us to conclude that a domain $S_{n}(D)$ is Kobayashi complete if $D \subset \mathbb{C}$ is bounded. In the unbounded case we should be more careful. Below we present a complete description of Kobayashi hyperbolicity and completeness of symmetric products of planar domains. We start with the special case.

Proposition 15 Fix $n, N \geq 2$. Let $\mu_{1}, \ldots, \mu_{N} \in \mathbb{C}$ be pairwise different.

If $N \geq 2 n$ then the domain $S_{n}\left(\mathbb{C} \backslash\left\{\mu_{1}, \ldots, \mu_{N}\right\}\right)$ is Kobayashi complete.

If $N<2 n$ then the domain $S_{n}\left(\mathbb{C} \backslash\left\{\mu_{1}, \ldots, \mu_{N}\right\}\right.$ contains a non-constant holomorphic image of $\mathbb{C}$ and thus it is not Kobayashi hyperbolic.

Proof Simple calculations give the following equality

$$
S_{n}\left(\mathbb{C} \backslash\left\{\mu_{1}, \ldots, \mu_{N}\right\}\right)=\mathbb{C}^{n} \backslash \bigcup_{j=1}^{N} H_{j},
$$

where (compare Proposition 9)

$$
\begin{aligned}
& H_{j} \\
& \quad:=\left\{\left(\mu_{j}+\pi_{n-1,1}(\lambda), \mu_{j} \pi_{n-1,1}(\lambda)+\pi_{n-1,2}(\lambda), \ldots, \mu_{j} \pi_{n-1, n-1}(\lambda)\right): \lambda \in \mathbb{C}^{n-1}\right\} \\
& =\left\{\left(\mu_{j}+z_{1}, \mu_{j} z_{1}+z_{2}, \ldots, \mu_{j} z_{n-1}\right):\left(z_{1}, \ldots, z_{n-1}\right) \in \mathbb{C}^{n-1}\right\}
\end{aligned}
$$

$j=1, \ldots, n-1$. Note that the hyperplanes $H_{j}$ are in general position. In fact, for any $1 \leq j_{1}<\cdots<j_{k} \leq n$ with $1 \leq k \leq N$ we get that

$$
H_{j_{1}} \cap \cdots \cap H_{j_{k}}=\left\{\pi_{n}\left(\mu_{j_{1}}, \ldots, \mu_{j_{k}}, \lambda_{1}, \ldots, \lambda_{n-k}\right): \lambda_{l} \in \mathbb{C}\right\}
$$

is an $(n-k)$-dimensional affine space (see Remark 10).

Then the theorem on Kobayashi completeness of the complement of the unions of $(2 n+1)$ hyperplanes in general position in the projective space (see [8,11]) and results on non-hyperbolicity of complements of $2 n$ hyperplanes (see [10] and [17] or [11]) finish the proof.

Theorem 16 Let $D \subset \mathbb{C}$ be a domain and let $n \geq 2$ be fixed. If $\#(\mathbb{C} \backslash D) \geq 2 n$ then $S_{n}(D)$ is Kobayashi complete. If $\#(\mathbb{C} \backslash D)<2 n$ then $S_{n}(D)$ contains a non-constant holomorphic image of $\mathbb{C}$ and thus $S_{n}(D)$ is not Kobayashi hyperbolic.

Proof In view of the previous result it is sufficient to show the first part of the theorem. Let $T \subset \mathbb{C} \backslash D$ be any set with $2 n$-elements. Then $S_{n}(D) \subset S_{n}(\mathbb{C} \backslash T)$ so the 
contractivity of the Kobayashi pseudodistance implies that $k_{S_{n}(D)} \geq k_{S_{n}(\mathbb{C} \backslash T)}$, which together with the previous result implies the Kobayashi hyperbolicity of $S_{n}(D)$. To prove the Kobayashi completeness it is sufficient to show that $k_{S_{n}(D)}\left(z^{1}, z^{k}\right) \rightarrow \infty$ for any sequence $\left(z^{k}\right)_{k} \subset D$ such that $\left\|z^{k}\right\| \rightarrow \infty$ or $z^{k} \rightarrow z^{0} \in \partial S_{n}(D)$. In the first case the result follows from Proposition 15 (as $k_{S_{n}(\mathbb{C} \backslash T)}\left(z^{1}, z^{k}\right) \rightarrow \infty$ ). In the case $z^{k} \rightarrow z^{0}=\pi_{n}\left(\mu_{1}, \ldots \mu_{n}\right)$, where $\mu_{1} \in \partial D, \mu_{j} \in \bar{D}, j=2, \ldots, n$ we choose a set $T \subset \bar{D}$ having $2 n$ elements such that $\mu_{j} \in T, j=1, \ldots, n$ which is possible due to the assumptions. Then $D \subset \mathbb{C} \backslash T$ and $z^{0} \in \partial S_{n}(\mathbb{C} \backslash T)$ so

$$
k_{S_{n}(D)}\left(z^{1}, z^{k}\right) \geq k_{S_{n}(\mathbb{C} \backslash T)}\left(z^{1}, z^{k}\right) .
$$

And the last expression tends to infinity by Proposition 15, which finishes the proof.

Remark 17 As we saw in the proof of Theorem 16 the description of Kobayashi complete symmetric products of planar domains relied not only on the linear convexity of $S_{n}(D)$ but also on the special geometry of $S_{n}(D)$. It could be interesting to see whether the following could be true: a linearly convex domain, which admits a certain number (at least $2 n$ ) of hyperplanes in a general position disjoint from the domain, is Kobayashi complete.

\subsection{Carathéodory Hyperbolicity}

It turns out that in the class of symmetric products of planar domains the Carathéodory hyperbolicity is preserved under taking symmetric powers.

Proposition 18 Let $D$ be a domain in $\mathbb{C}$. Then $D$ is Carathéodory hyperbolic if and only if $S_{n}(D)$ is Carathéodory hyperbolic. Consequently, $D$ is c-finitely compact if and only if $S_{n}(D)$ is c-finitely compact.

Proof It is sufficient to show that $c$-hyperbolicity of $D$ implies that of $S_{n}(D)$. Assume that $D$ is Carathéodory hyperbolic. Let $\pi_{n}\left(\lambda_{1}, \ldots, \lambda_{m}\right) \neq \pi_{n}\left(\mu_{1}, \ldots, \mu_{n}\right)$ with $\lambda_{j}, \mu_{j} \in D, j=1, \ldots, n$. Then without loss of generality we may assume that $\lambda_{1} \notin\left\{\mu_{1}, \ldots, \mu_{n}\right\}=\left\{x_{1}, \ldots, x_{k}\right\}$. Then we claim that there is an $f \in \mathcal{O}(D, \mathbb{D})$ with $f\left(\lambda_{1}\right)=0, f\left(\mu_{j}\right) \neq 0$. In fact, the Carathéodory hyperbolicity implies that there is a non-constant bounded $g \in \mathcal{O}(D)$ such that $g\left(\lambda_{1}\right)=0$. Then the function $h(\cdot):=\frac{g(\cdot)}{\prod_{j=1}^{k}\left(\cdot-x_{j}\right)^{r_{j}}}$, where $r_{j}$ is the multiplicity of $g$ at $x_{j}$, is a bounded holomorphic function with $h\left(\lambda_{1}\right)=0$ and $h\left(\mu_{j}\right) \neq 0$ which gives the claim. Take the function $f$ from the claim. Then $z:=\pi_{n}\left(f\left(\lambda_{1}\right), \ldots, f\left(\lambda_{n}\right)\right) \neq \pi_{n}\left(f\left(\mu_{1}\right), \ldots, f\left(\mu_{n}\right)\right)=: w$, so

$$
c_{S_{n}(D)}\left(\pi_{n}\left(\lambda_{1}, \ldots, \lambda_{n}\right), \pi_{n}\left(\mu_{1}, \ldots, \mu_{n}\right)\right) \geq c_{\mathbb{G}_{n}}(z, w)>0 .
$$

Remark 19 Recall that in the class of planar domains by a recent result (Theorem 1 in [6]) two closely related notions of Carathéodory completeness and $c$-finite compactness are equivalent. Moreover, they are both equivalent to the fact that any boundary 
point $z$ of $D$ is an $A(D,\{z\})$ peak point. Note that although the $c$-finite compactness is equivalent to $c$-finite compactness of $S_{n}(D)$ (Proposition 18) we did not prove the equivalence of $c$-finite compactness of $S_{n}(D)$ with the fact that any boundary point $z$ of $S_{n}(D)$ is a weak $A(D,\{z\})$ point.

Acknowledgements The author was partially supported by the OPUS Grant No. 2015/17/B/ST1/00996 financed by the National Science Centre, Poland.

Open Access This article is licensed under a Creative Commons Attribution 4.0 International License, which permits use, sharing, adaptation, distribution and reproduction in any medium or format, as long as you give appropriate credit to the original author(s) and the source, provide a link to the Creative Commons licence, and indicate if changes were made. The images or other third party material in this article are included in the article's Creative Commons licence, unless indicated otherwise in a credit line to the material. If material is not included in the article's Creative Commons licence and your intended use is not permitted by statutory regulation or exceeds the permitted use, you will need to obtain permission directly from the copyright holder. To view a copy of this licence, visit http://creativecommons.org/licenses/by/4.0/.

\section{References}

1. Agler, J., Young, N.J.: The hyperbolic geometry of the symmetrised bidisc. J. Geom. Anal. 14, 375-403 (2004)

2. Bharali, G., Biswas, I., Divakaran, D., Janardhanan, J.: Proper holomorphic mappings onto symmetric products of a Riemann surface. Doc. Math. 23, 1291-1311 (2018)

3. Chakrabrati, D., Gorai, S.: Function theory and holomorphic maps on symmetric products of planar domains. J. Geom. Anal. 25(4), 2196-2225 (2015)

4. Chakrabarti, D., Grow, C.: Proper holomorphic self-maps of symmetric powers of balls. Arch. Math. 110, 45-52 (2018)

5. Costara, C.: Lempert's theorem and the symmetrized bidisc. Bull. Lond. Math. Soc. 36(5), 656-662 (2004)

6. Edigarian, A.: Carathéodory completeness on the plane. (2018). arXiv:1803.08714

7. Edigarian, A., Zwonek, W.: Geometry of the symmetrized polydisc. Arch. Math. (Basel) 84, 364-374 (2005)

8. Green, M.L.: The hyperbolicity of the complement of $2 n+1$ hyperplanes in general position in $P_{n}$, and related results. Proc. Am. Math. Soc. 66(1), 109-113 (1977)

9. Jarnicki, M., Pflug, P.: Invariant Distances and Metrics in Complex Analysis, de Gruyter, 2nd extended edition (2013)

10. Kiernan, P.: Hyperbolic submanifolds of complex projective space. Proc. Am. Math. Soc. 22, 603-606 (1969)

11. Kobayashi, S.: Hyperbolic Complex Spaces. Grundlehre d. mathematischen Wissenschaften, vol. 318 (1998)

12. Kosiński, Ł., Zwonek, W.: Proper holomorphic mappings vs. peak points and Shilov boundary. Ann. Polon. Math. 107, 97-108 (2013)

13. Lempert, L.: La métrique de Kobayashi et la représentation des domaines sur la boule. Bull. Soc. Math. Fr. 109, 427-474 (1981)

14. Nikolov, N., Pflug, P., Zwonek, W.: The Lempert function of the symmetrized polydisc in higher dimensions is not a distance. Proc. Am. Math. Soc. 135(9), 2921-2928 (2007)

15. Nikolov, N., Pflug, P., Zwonek, W.: An example of a bounded $\mathbb{C}$-convex domain which is not biholomorphic to a convex domain. Math. Scand. 102(1), 149-155 (2008)

16. Nikolov, N., Trybuła, M.: Gromov hyperbolicity of the Kobayashi metric on "convex" sets. J. Math. Anal. Appl. 468(2), 1164-1178 (2018)

17. Snurnitsyn, V.E.: The complement of $2 n$ hyperplanes in $C P^{n}$ is not hyperbolic. Mat. Zametki 40 , 455-459 (1986) 
18. Whitney, H.: Complex Analytic Varieties. Addison-Wesley Series in Mathematics. Addison-Wesley Publishing Co., Reading, London (1972)

Publisher's Note Springer Nature remains neutral with regard to jurisdictional claims in published maps and institutional affiliations. 